This item is the archived peer-reviewed author-version of:

\title{
Circular motion analysis of time-varying bioimpedance
}

\section{Reference:}

Sanchez B., Louarroudi Ebrahim, Rutkove S.B., Pintelon R..- Circular motion analysis of time-varying bioimpedance Physiological measurement / Institute of Physical Sciences in Medicine - ISSN 0967-3334 - 36:11(2015), p. 2353-2367 Full text (Publishers DOI): http://dx.doi.org/10.1088/0967-3334/36/11/2353

Full text (Publishers DOI): http://dx.doi.org/doi:10.1088/0967-3334/36/11/2353

To cite this reference: http://hdl.handle.net/10067/1284220151162165141 


\title{
Circular motion analysis of time-varying bioimpedance
}

\author{
B Sanchez ${ }^{1} \ddagger$ E Louarroudi ${ }^{2}$, S B Rutkove ${ }^{1}$ and R Pintelon ${ }^{3}$ \\ ${ }^{1}$ Department of Neurology, Division of Neuromuscular Diseases, Beth Israel \\ Deaconess Medical Center, Harvard Medical School, Boston, MA 02215-5491, USA. \\ ${ }^{2}$ Optical Metrology, 3D Design and Mechanics (Op3Mech) Research Group, Faculty \\ of Applied Engineering, University of Antwerp, 2660 Antwerp, Belgium. \\ ${ }^{3}$ Department of Fundamental Electricity (ELEC), Vrije Universiteit Brussel (VUB), \\ B-1050, Brussels, Belgium. \\ E-mail: bsanchez@bidmc.harvard.edu and ebrahim.louarroudi@uantwerpen.be
}

\begin{abstract}
This paper presents a step forward towards the analysis of a linear periodically time-varying (PTV) bioimpedance $Z_{\mathrm{PTV}}(j \omega, t)$, which is an important subclass of a linear time-varying (LTV) bioimpedance. Similarly to the Fourier coefficients of a periodic signal, a PTV impedance can be decomposed into frequency dependent impedance phasors, $Z_{r}(j \omega) e^{j 2 \pi \frac{r}{T_{\mathrm{Z}}} t}, r \in \mathbb{Z}_{0}$, that are rotating with an angular speed of $\varpi_{r}=2 \pi r / T_{\mathrm{Z}}$. The vector length of these impedance phasors corresponds to the amplitude of the $r$ th-order harmonic impedance $\left|Z_{r}(j \omega)\right|$ and the initial phase is given by $\Phi_{r}\left(\omega, t_{0}\right)=\angle Z_{r}(j \omega)+2 \pi r t_{0} / T_{\mathrm{Z}}$, with $t_{0} \in[0, T]$ being a time instant within the measurement time $T$. The impedance period $T_{\mathrm{Z}}$ stands for the cycle length of the bio-system under investigation; for example, the elapsed time between two consecutive $\mathrm{R}$-waves in the electrocardiogram or the breathing periodicity in case of the heart or lungs, respectively. First, it is demonstrated that the harmonic impedance phasor $Z_{r}\left(j \omega_{k}\right) e^{j 2 \pi \frac{r}{T_{Z}} t}$, at a particular measured frequency $\omega_{k}$, can be represented by a rotating phasor, leading to the so-called circular motion analysis technique. Next, the two-dimensional (2-D) representation of the harmonic impedance phasors is then extended to a three-dimensional (3-D) coordinate system by taking into account the frequency dependence. Finally, we introduce a new visualizing tool to summarize the frequency response behavior of $Z_{\mathrm{PTV}}(j \omega, t)$ into a single 3-D plot using the local Frenet-Serret frame. This novel 3-D impedance representation is then compared with the 3-D Nyquist representation of a PTV impedance. The concepts are illustrated through real measurements conducted on a PTV $R C$-circuit.
\end{abstract}

Keywords: (periodically) time-varying impedance [(P)TV], best linear time-invariant (BLTI) impedance, harmonic impedance, impedance spectroscopy (IS), multisine

‡ Corresponding authors: Dr. Benjamin Sanchez, Department of Neurology, Division of Neuromuscular Diseases, Beth Israel Deaconess Medical Center, Harvard Medical School, 330 Brookline Avenue, Boston, MA 02215-5491, USA. Phone: +1 617667 3057. Dr. Ebrahim Louarroudi, Optical Metrology, 3D design and Mechanics (Op3Mech) group, Faculty of Applied Engineering, University of Antwerp, Salesianenlaan 30, 2660 Antwerp, Belgium. Phone: +32 (0)3 2051841 
excitations.

\section{Introduction}

The convenience of the linearity and time invariance (LTI) assumptions when measuring an impedance, i.e. $Z_{\mathrm{LTI}}(j \omega)$, has been proven undeniable by the extensive literature on the topic (Barsoukov \& Macdonald 2005, Grimnes \& Martinsen 2014). Likewise, it is also irrefutable that many bio-systems are time-variant. Interestingly, in spite of many bio-systems being time-variant, the process of finding the frequency response of these systems using impedance spectroscopy (IS) has relied historically on methods and models intended for systems with an invariant behavior over time. This observation was already perceived by (Eisenberg 1980, p. 320) when he noted that it was not yet possible to perform IS to study the contraction of skeletal muscle. Another example is impedance cardiography where the time-variant impedance is measured at one single frequency using LTI methods (Kubicek et al. 1966). A review of the literature shows that there is no clear consensus on the optimal measurement frequency (Bernstein \& Lemmens 2005), typically chosen from 20 to $100 \mathrm{kHz}$ (Sherwood et al. 1990). Furthermore, it remains unknown how the error at the measurement frequency affects stroke volume determinations. Those two questions are just an example of why it is so important to develop more sophisticated methods to study time-variant bio-systems using IS.

The theory for time-variant systems (Zadeh 1950b, Zadeh 1950a) has been applied in all kind of engineering applications. Recent examples using IS are available in the study of metal corrosion (Van Ingelgem et al. 2008), the determination of the stateof-charge of batteries (Pop et al. 2005), and the measurement of in vivo myocardial impedance (Sanchez et al. 2013a, Sanchez et al. 2013b). Algorithms for modeling such systems can be classified according to the parametrization used, i.e. parametric in both the dynamics and time (Tóth et al. 2012, Louarroudi et al. 2013), parametric in time and nonparametric in the dynamics (Sams \& Marmarelis 1988, Louarroudi et al. 2012, Pintelon et al. 2015), parametric in the dynamics and nonparametric in time (Niedzwiecki \& Kaczmarek 2005), nonparametric in both the dynamics and time (Allen \& Rabiner 1977), and references therein.

This paper presents a step further towards the analysis of linear time-varying (LTV) impedance $Z_{\mathrm{LTV}}(j \omega, t)$ with a dominant periodic behavior, denoted as periodically timevarying $(\mathrm{PTV})$ impedance $Z_{\mathrm{PTV}}(j \omega, t)$. The remainder of this paper is organized as follows. Section 2 recalls the harmonic impedance concept associated with $Z_{\mathrm{PTV}}(j \omega, t)$. Based on impedance phasors, we present in section 2 the two- and three-dimensional (2/3-D) circular motion analysis technique of $Z_{\mathrm{PTV}}(j \omega, t)$. Section 3 extends the analysis of $Z_{\mathrm{PTV}}(j \omega, t)$ in a 3-D coordinate system using the Frenet-Serret local frame. Afterwards in section 4, the Frennet-Serret approach is compared with the 3-D Nyquist representation of a PTV impedance. The new concepts are illustrated on measurements originating from a PTV $R C$-circuit in section 5-6. The main findings are discussed in 
section 7 and summarized under the form of conclusions in section 8 .

\section{Circular motion analysis of periodically time-varying impedance}

An impedance is called periodically time-varying (PTV) if the impedance can be written under the form (Sanchez et al. 2015)

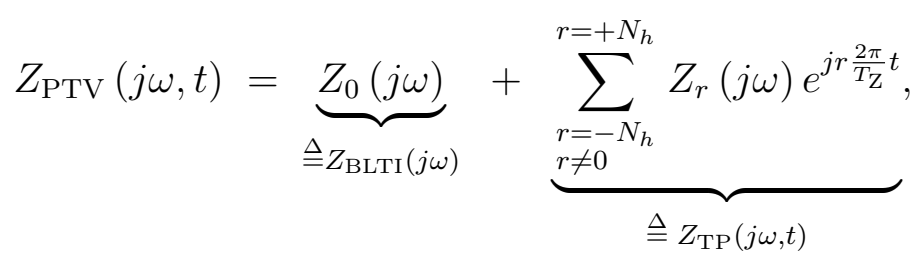

where $Z_{r}(j \omega)$ are known as the harmonic impedance $\left(N_{h}\right.$ harmonics $)$ and with $T_{\mathrm{Z}}$ being the periodicity of the impedance, i.e. $Z_{\mathrm{PTV}}\left(j \omega, t+T_{\mathrm{Z}}\right)=Z_{\mathrm{PTV}}(j \omega, t)$. The number of significant harmonics $N_{h}$ depends on the strength of the temporal variation in $Z_{\mathrm{PTV}}(j \omega, t)$. To obtain in practice the correct order $N_{h}$, it is recommended to examine each individual skirt component in the output voltage spectrum (Sanchez et al. 2013a).

\subsection{2-D impedance phasor representation}

First, notice from $(1)$ that $Z_{\mathrm{PTV}}(j \omega, t)$ can be separated into two main contributions.

- The first term in the right-hand side of (1) is the zero-order harmonic contribution $Z_{0}(j \omega)$. More specifically, it has been shown in (Louarroudi et al. 2014) that $Z_{0}(j \omega)$ equals the best - in a mean square sense - linear time-invariant (BLTI) impedance approximation of $Z_{\mathrm{PTV}}(j \omega, t)$, which is defined as the mean impedance

$$
Z_{\mathrm{BLTI}}(j \omega) \triangleq \frac{1}{T_{\mathrm{Z}}} \int_{t_{0}}^{t_{0}+T_{\mathrm{Z}}} Z_{\mathrm{PTV}}(j \omega, t) d t,
$$

with $t_{0}$ being an arbitrary time instant within the measurement interval. The geometrical representation of $Z_{\mathrm{BLTI}}(j \omega)$ (2) at one particular frequency $\omega=\omega_{k}$ in the complex plane is a static (time-invariant) impedance vector (see figure $1(\mathrm{a})$ ). The projections of $Z_{\mathrm{BLTI}}\left(j \omega_{k}\right)(2)$ on the $x$ and $y$ axes in figure 1 (a) are the resistive and reactive components of $Z_{\text {BLTI }}\left(j \omega_{k}\right)$ (Barsoukov \& Macdonald 2005, Grimnes \& Martinsen 2014).

- The second part in the right-hand side of (1) is called the time-periodic (TP) part $Z_{\mathrm{TP}}(j \omega, t)$, which contains all the contributions of the higher order harmonic impedances $Z_{r}(j \omega)$. As shown in figure 1(b), when each harmonic component at

$\omega=\omega_{k}$, i.e. $Z_{r}\left(j \omega_{k}\right) e^{j 2 \pi \frac{r}{T_{\mathrm{Z}}} t}, r \neq 0$, is analyzed separately, one can see that it acts as an impedance phasor which is rotating at an angular speed of $\varpi_{r}=2 \pi r / T_{\mathrm{Z}}$ with

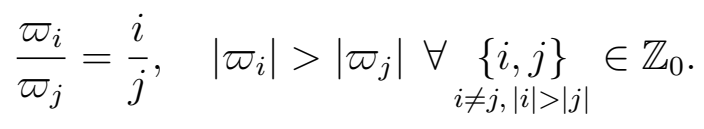


The direction of rotation is counterclockwise for $r \in \mathbb{Z}^{+}$and clockwise for $r \in \mathbb{Z}^{-}$. The phasor's instantaneous phase is $\Phi_{r}\left(\omega_{k}, t_{0}\right)=\angle Z_{r}\left(j \omega_{k}\right)+2 \pi r t_{0} / T_{\mathrm{Z}}$ with $t_{0}$ a time instant within the measurement time $T$.

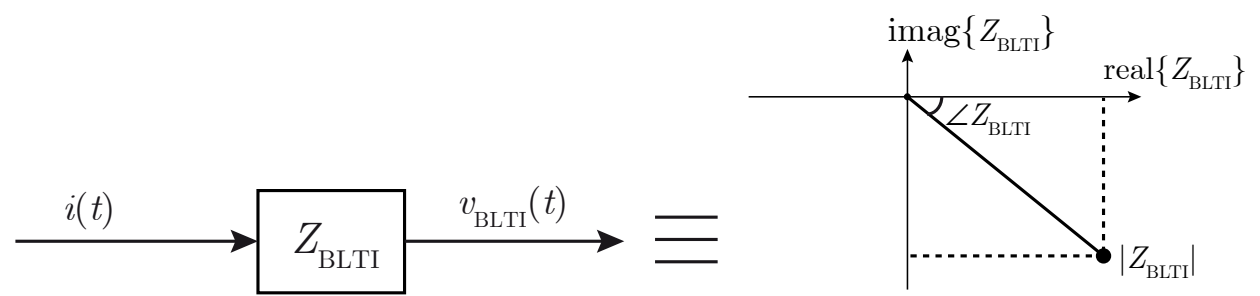

(a) Schematic of the static impedance phasor corresponding to the best linear timeinvariant (BLTI) impedance approximation $Z_{\mathrm{BLTI}}\left(j \omega_{k}\right)$ in (1).

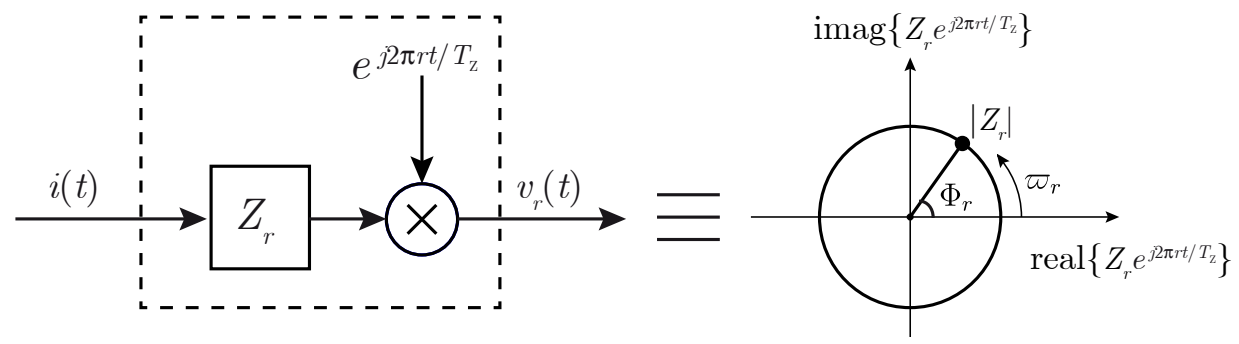

(b) Schematic of the rotating impedance phasor corresponding to the $r$ th harmonic of $Z_{\mathrm{TP}}\left(j \omega_{k}, t\right)$ in (1). The length of the phasor (radius) is determined by the amplitude of the $r$ th-order harmonic impedance $\left|Z_{r}\left(j \omega_{k}\right)\right|, r \in \mathbb{Z}^{ \pm}$. The black dot on top of the circle represents the initial phase $\Phi_{r}\left(\omega_{k}, t_{0}\right)$ at $t_{0}$. The angular velocity is $\varpi_{r}$ and the direction of rotation, denoted by the black arrow, is counterclockwise for $r \in \mathbb{Z}^{+}$and clockwise for $r \in \mathbb{Z}^{-}$.

Figure 1. Two-dimensional geometrical interpretation of a periodically time-varying (PTV) impedance $Z_{\mathrm{PTV}}\left(j \omega_{k}, t\right)$ by means of (a) the best linear time-invariant (BLTI) impedance approximation $Z_{\mathrm{BLTI}}\left(j \omega_{k}\right)$, and (b) the circular motion decomposition of $Z_{\mathrm{TP}}\left(j \omega_{k}, t\right) . T_{\mathrm{Z}}$ denotes the impedance period and $t_{0} \in[0, T]$ is a relative time instant within the measurement time $T$.

Adding up the individual contribution of the static $\left(Z_{\mathrm{BLTI}}\left(j \omega_{k}\right)\right)$ and the rotating phasors $\left(Z_{r}\left(j \omega_{k}\right), r \neq 0\right)$ finally defines the periodic orbit of $Z_{\mathrm{PTV}}\left(j \omega_{k}, t\right)$ in the complex plane, as shown schematically in figure 2 (bottom right).

\subsection{3-D impedance phasor representation}

Until now, the analysis in figure 1 and figure 2 is done at one particular measured frequency $\omega_{k}$. However, in case different frequencies are measured, i.e. performing IS, the frequency dependence should be included by adding a new axis. Figure 3 shows such a conceptualized 3-D representation for one particular harmonic impedance phasor, $Z_{r}\left(j \omega_{k}\right) e^{j 2 \pi \frac{r}{T_{\mathrm{Z}}} t}, r \neq 0$, with $k=1,2, \ldots, F$ and $F$ being the number of measured frequencies. One can see that it results in a kind of a "horn-shaped" surface made of $k=1,2, \ldots, F$ different rotating impedance phasors with varying radii and initial phases. 


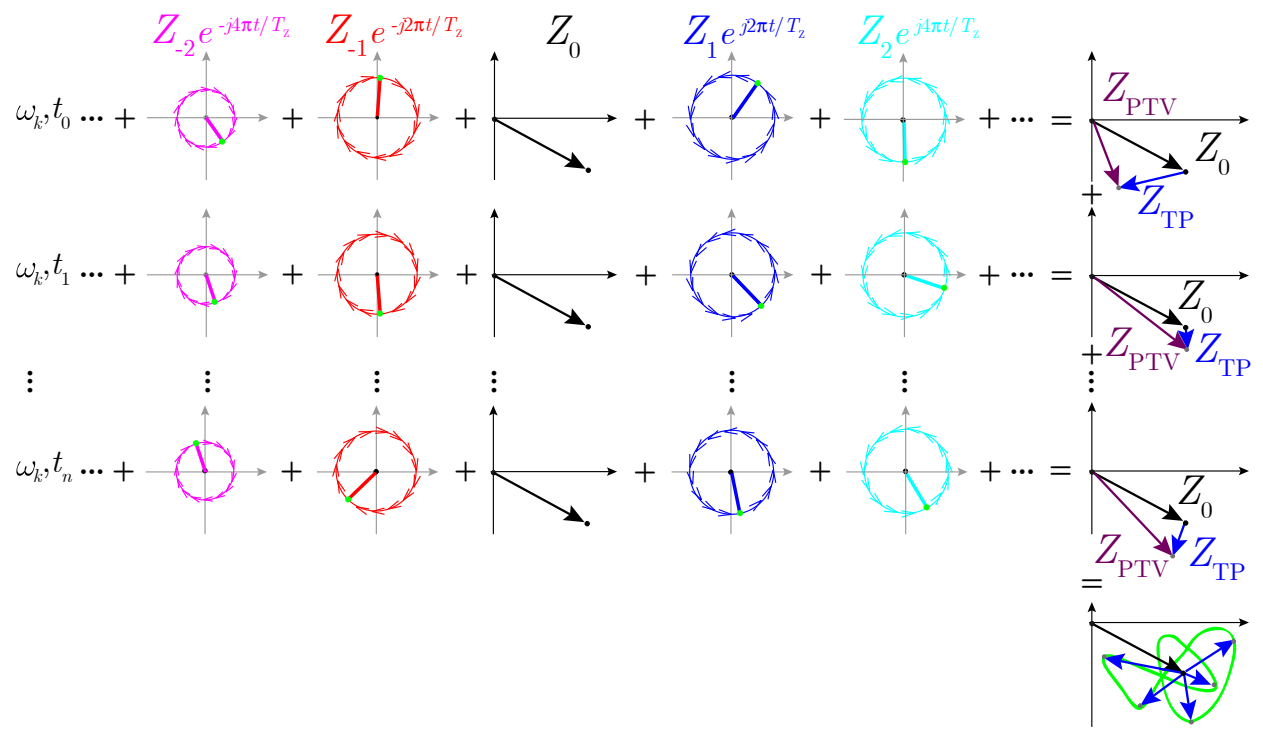

Figure 2. Circular motion decomposition of periodically time-varying (PTV) impedance $Z_{\mathrm{PTV}}\left(j \omega_{k}, t\right)$. At one particular frequency $\omega_{k}$ and at each continuous time instant $t_{n}$, the geometric sum of the rotating phasors defines the time-periodic (TP) impedance $Z_{\mathrm{TP}}\left(j \omega_{k}, t\right)$ in (2) (blue arrows, right column). The black and dark red arrows are, respectively, the best linear time-invariant (BLTI) impedance approximation $Z_{\mathrm{BLTI}}\left(j \omega_{k}\right)$ and $Z_{\mathrm{PTV}}\left(j \omega_{k}, t\right)$. Note that the black and gray colors in the $x$ and $y$ axes show the differences between coordinate systems in figure 1(a)-1(b).

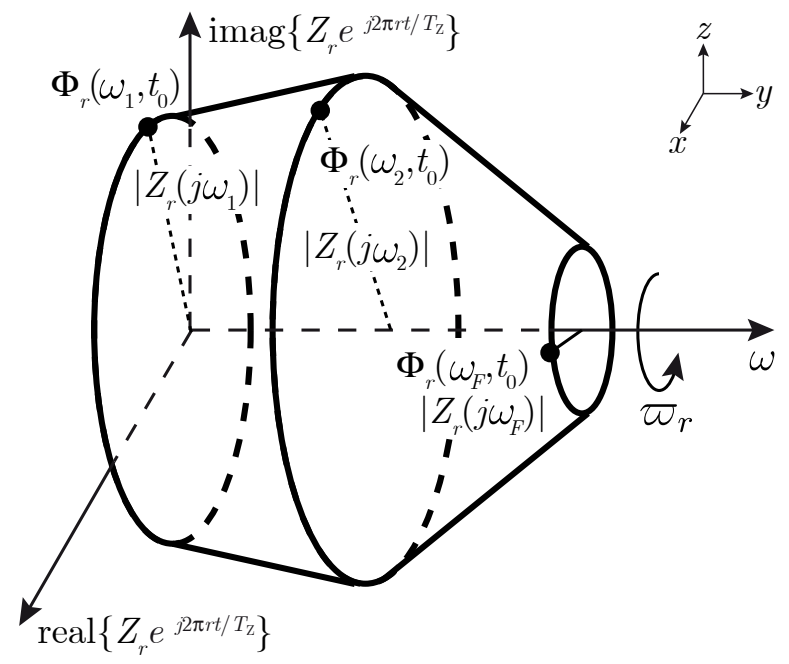

Figure 3. Schematic illustrating the three-dimensional (3-D) geometric interpretation of the two-dimensional harmonic impedance phasors representation in figure 1 (b). The 3 -D representation results in a kind of horn-shaped surface made of $\left|Z_{r}\left(j \omega_{k}\right)\right|$ and the initial phase $\Phi_{r}\left(\omega_{k}, t_{0}\right)$ for $k=1,2, \ldots, F$, with $F$ being the number of measured frequencies. The angular speed of the phasors is $\varpi_{r}$ and the direction of rotation, denoted by the black arrow, is counterclockwise for $r \in \mathbb{Z}^{+}$and clockwise for $r \in \mathbb{Z}^{-}$. 


\section{3-D PTV impedance representation in the Frenet-Serret frame}

To reconstruct a 3-D visualizing tool for periodically varying bio-systems, we assume that the BLTI impedance arc $\vec{Z}_{0}:\left[\omega_{\min }, \omega_{\max }\right] \subset \mathbb{R} \rightarrow \mathbb{R}^{3}$

$$
\vec{Z}_{0}(\omega)=\left(\begin{array}{c}
0 \\
\operatorname{real}\left\{Z_{0}(j \omega)\right\} \\
\operatorname{imag}\left\{Z_{0}(j \omega)\right\}
\end{array}\right) \text { for } \omega \in\left[\omega_{\min }, \omega_{\text {max }}\right] \subset \mathbb{R}
$$

is differentiable in $\mathbb{R}^{3}$ such that the tangent $\vec{t}$, normal $\vec{n}$ and binormal $\vec{b}$ unit vectors

$$
\vec{t}=\vec{n} \times \vec{b}, \quad \vec{n}=\vec{b} \times \vec{t}, \quad \vec{b}=\vec{t} \times \vec{n}
$$

associated with the BLTI impedance arc in figure 4 can be computed. Note that, for convenience, the $x$-component of the BLTI impedance arc (3) is chosen to be zero such that the arc is located in the $y z$-plane (shown in gray in figure 4 ).

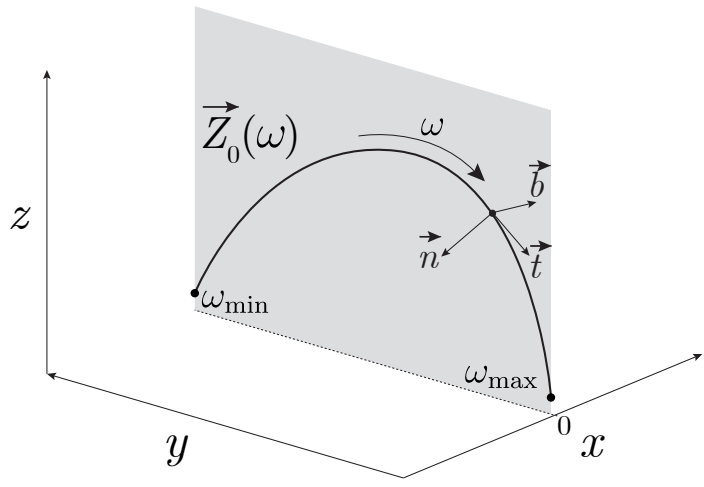

Figure 4. Three-dimensional schematic representation of the best linear timeinvariant (BLTI) impedance arc $\vec{Z}_{0}:\left[\omega_{\min }, \omega_{\max }\right] \subset \mathbb{R} \rightarrow \mathbb{R}^{3}(3)$ in the $y z$-plane (in gray at $x=0$ ). The black dots indicate the minimum and maximum frequency, $\omega_{\min }$ and $\omega_{\max }$ respectively. The vectors $\vec{t}, \vec{n}, \vec{b}$ represent the tangent, normal, and binormal unit vectors of the Frenet-Serret frame at one particular frequency $\omega$.

Since in practice the arc (3) in figure 4 is only measured at a discrete set of frequencies $\omega_{k}$ for $k=1,2, \ldots, F$, we can define the "discrete version" of (3) as the matrix $\mathbf{Z}_{0}$ built with the real and imaginary parts of $Z_{0}\left(j \omega_{k}\right)$ for $k=1,2, \ldots, F$, namely

$$
\mathbf{Z}_{0}=\left[\begin{array}{ccccc}
0 & \cdots & 0 & \cdots & 0 \\
\operatorname{real}\left\{Z_{0}\left(j \omega_{1}\right)\right\} & \cdots & \operatorname{real}\left\{Z_{0}\left(j \omega_{k}\right)\right\} & \cdots & \operatorname{real}\left\{Z_{0}\left(j \omega_{F}\right)\right\} \\
\operatorname{imag}\left\{Z_{0}\left(j \omega_{1}\right)\right\} & \cdots & \operatorname{imag}\left\{Z_{0}\left(j \omega_{k}\right)\right\} & \cdots & \operatorname{imag}\left\{Z_{0}\left(j \omega_{F}\right)\right\}
\end{array}\right] .
$$

According to the Frenet-Serret approach (Serret 1851, Frenet 1852), the tangent, normal and binormal unit vectors in (4), can be computed (numerically) from the $k$ 
columns of $\mathbf{Z}_{0}$, i.e. $\mathbf{Z}_{0[:, k]}$ for $k=1,2, \ldots, F$ frequencies, as follows

$$
\vec{t}_{[k]}=\left(0, y_{[k]}, z_{[k]}\right)^{T} \approx \frac{\Delta\left\{\mathbf{Z}_{0[:, k]}\right\}}{\left\|\Delta\left\{\mathbf{Z}_{0[:, k]}\right\}\right\|_{2}}, \quad \vec{n}_{[k]}=\frac{\left(0, z_{[k]},-y_{[k]}\right)^{T}}{\sqrt{y_{[k]}^{2}+z_{[k]}^{2}}}, \quad \vec{b}_{[k]}=\vec{t}_{[k]} \times \vec{n}_{[k]},
$$

with $(\bullet)^{T}$ being the vector transpose, $\Delta[\bullet]$ being the central difference operator

$$
\Delta\left\{\mathbf{Z}_{0[:, k]}\right\}=\left\{\begin{array}{lll}
\mathbf{Z}_{0[:, k+1]}-\mathbf{Z}_{0[:, 1]} & \text { if } & k=1 \\
\mathbf{Z}_{0[:, k+1]}-\mathbf{Z}_{0[:, k-1]} & \text { if } & 1<k<F \\
\mathbf{Z}_{0[:, k]}-\mathbf{Z}_{0[:, k-1]} & \text { if } & k=F
\end{array}\right.
$$

and $\|\bullet\|_{2}$ denotes the vector norm

$$
\|\vec{x}\|_{2}=\sqrt{x^{2}+y^{2}+z^{2}}
$$

Then, the matrix containing the $r$ th-order harmonic impedance phasors, $\mathbf{Z}_{r}(t)$ with $t \in\left[t_{0}, t_{0}+T_{Z}\right]$, can readily be found in the Frenet-Serret local frame as

$$
\mathbf{Z}_{r[:, k]}(t)=\left|Z_{r}\left(j \omega_{k}\right)\right| \cdot\left[\vec{n}_{[k]} \cdot \cos \left(\Phi_{r}\left(\omega_{k}, t\right)\right)+\vec{b}_{[k]} \cdot \sin \left(\Phi_{r}\left(\omega_{k}, t\right)\right)\right] .
$$

The last step is to represent $\mathbf{Z}_{\mathrm{PTV}}(t)$ in the Frenet-Serret frame by combining $\mathbf{Z}_{0}$ in (5) and $\mathbf{Z}_{r}(t)$ in $(9)$ as follows

$$
\mathbf{Z}_{\mathrm{PTV}}(t)=\mathbf{Z}_{0}+\sum_{\substack{r=-N_{h} \\ r \neq 0}}^{+N_{h}} \mathbf{Z}_{r}(t)
$$

Note that the curvature of the 3-D plot, described by expression (10), is determined by the $\operatorname{arc} \mathbf{Z}_{0}(5)$ (solid line in figure 4). This is in accordance with the 2-D Nyquist representation of a linear time-invariant impedance $Z_{\mathrm{LTI}}(j \omega)$. On top of the arc, the time-periodic impedance, $Z_{\mathrm{TP}}(j \omega, t)$ in $(1)$, is included in the plot as a sum of rotating phasors $\mathbf{Z}_{r}(t), r \neq 0$, projected in the Frenet-Serret local frame $(\{\vec{n}, \vec{b}\}-$ plane $)$. As explained in the next section, this 3 -D visualizing tool differs fundamentally from the 3-D Nyquist representation of a PTV impedance.

\section{3-D Nyquist representation}

An alternative to the new 3-D visualization method in section 3 is to represent the real and imaginary parts of the PTV impedance $Z_{\mathrm{PTV}}(j \omega, t)$ directly in a $3-\mathrm{D}$ Nyquist plot. The 3-D generalization of the 2-D Nyquist plot, which is applied to $Z_{\mathrm{PTV}}(j \omega, t)$, consists of adding the time dependence as a new axis (note that this representation is also valid for an arbitrarily time-varying impedance). The result, shown in figure 5, is the instantaneous impedance arc at each time instant $t_{n}, n \in \mathbb{N}$ provided that: 
(i) All the excited frequencies were measured at the same time instant, e.g. using multisine signals as in (Searle \& Kirkup 1999).

(ii) The PTV impedance measured at time instant $t_{n}$, i.e. $Z_{\mathrm{PTV}}\left(j \omega, t_{n}\right)$, can be approximated as a time-invariant one within the measurement interval $T_{n}$, i.e. $Z_{\mathrm{LTI}}^{[n]}(j \omega) \approx Z_{\mathrm{PTV}}(j \omega, t)$ for $t \in\left[t_{n}-\frac{T_{n}}{2}, t_{n}+\frac{T_{n}}{2}\right]$.

If Assumption (i) is violated, for example, because $Z_{\mathrm{PTV}}(j \omega, t)$ was measured using the stepped-sine IS approach, then each impedance point would be located in a different temporal plane. Furthermore, if Assumption (ii) is not fulfilled, the impedance arc will be distorted. The reader can find an example in (Sanchez et al. 2015) of a 3-D Nyquist impedance arc measured with the stepped-sine approach which is distorted due to the time dependence of the impedance (see figure 10 (b) therein).

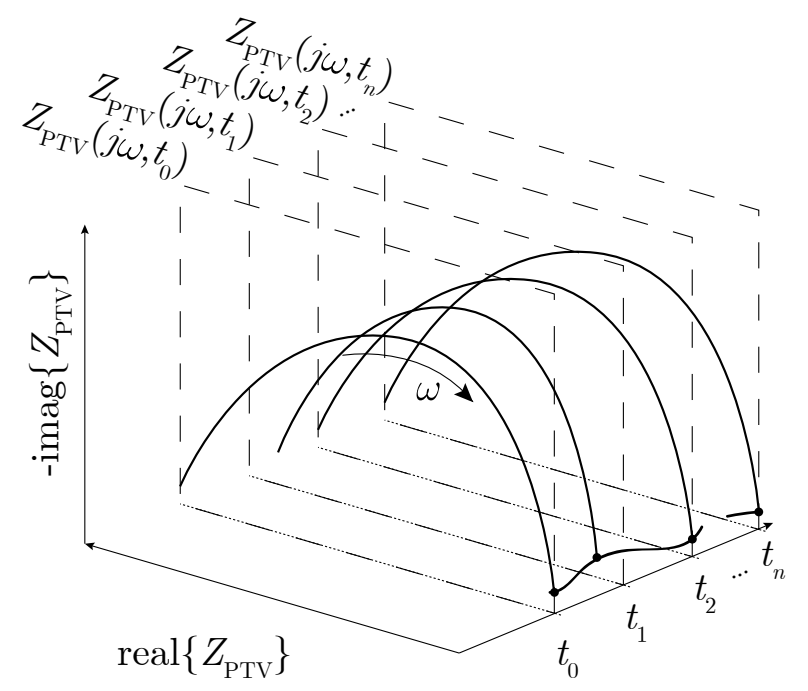

Figure 5. Three-dimensional Nyquist representation of a linear periodically timevarying $(\mathrm{PTV})$ impedance $Z_{\mathrm{PTV}}(j \omega, t)$ through instantaneous impedance arcs at discrete time instants $t_{n}, n \in \mathbb{N}$. In the figure, the frequencies corresponding to the same impedance arc are located in the same real and imaginary plane (limited by the dotted lines, e.g. e.g. measured with multisine excitations). The line connecting the dots illustrates the impedance evolution over time at one particular frequency $\omega$.

\section{An illustrative example}

Before delving into the measurement results, we first provide in section 5.1 an overview of the general aspects concerning the measurement and identification setup used.

\subsection{Brief description of the measurement and identification process}

We conducted a single 2-electrode spectroscopy experiment on the PTV $R C$-circuit shown in figure 6 using the parametric-in-time measurement strategy proposed in (Sanchez et al. 2013a). The noisy input-output signals coming from the voltage 


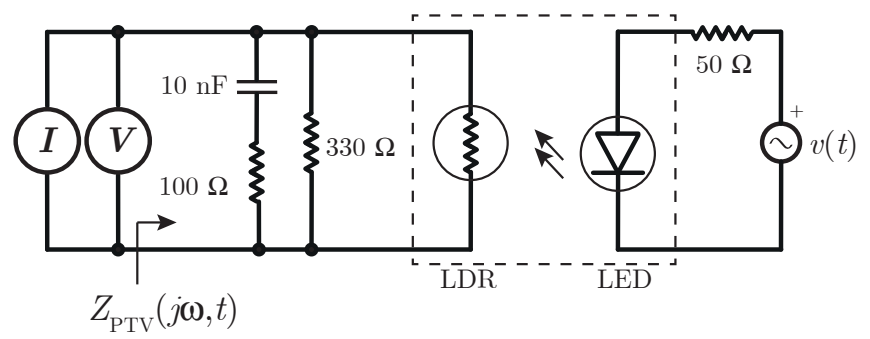

Figure 6. $R C$-circuit phantom emulating a periodically time-varying (PTV) impedance $Z_{\mathrm{PTV}}(j \omega, t)$. The current $I$ and voltage $V$ denote the terminals of the 2-electrode impedance measurement. The light emitting diode (LED) and the lightdependent resistor (LDR) were both covered to prevent impedance changes due to fluctuations of the ambient light. A LabVIEW program was used to generate a sinewave voltage scheduling signal $v(t)=V_{\mathrm{DC}}+\frac{V_{\mathrm{PP}}}{2} \sin \left(2 \pi f_{\mathrm{Z}} t\right)$ with settings: $V_{\mathrm{PP}}=0.1 \mathrm{~V}$ the peak-to-peak amplitude, $f_{\mathrm{Z}}=1 / T_{\mathrm{Z}}=1 \mathrm{~Hz}$ the frequency of the impedance cycle and $V_{\mathrm{DC}}=2.5 \mathrm{~V}$ the dc amplitude to set the LED into conduction.

controlled current source-based analogue front end $\left(1 \mathrm{~mA} \mathrm{~V}^{-1}\right)$ were acquired synchronously to the reference excitation and sampled at a constant sample rate of $f_{\mathrm{s}}=5 \mathrm{Ms} \mathrm{s}^{-1}$ (PXIe-5122, $100 \mathrm{Ms} \mathrm{s}^{-1}, 64 \mathrm{MB} /$ channel, $14 \mathrm{bits}$ ). The chosen reference signal was a random phase multisine signal with equal harmonic amplitudes (Sanchez et al. 2012) and generated with an arbitrary waveform generator (PXI-5421, $100 \mathrm{Ms} \mathrm{s}^{-1}$, $32 \mathrm{MB}, 16 \mathrm{bits})$. The fundamental period of the multisine was $1 \mathrm{~ms}$ and the phases of the reference signal were randomly distributed in $[0,2 \pi)$ (Pintelon \& Schoukens 2012). The multisine excitation consisted of $F=26$ frequencies quasi logarithmically distributed in the frequency band $1 \mathrm{kHz} \rightarrow 1 \mathrm{MHz}, f_{k}=\{1,3,5,7,11,15,19,25,33,41,51,63,77$, 95, 117, 143, 173, 173, 253, 307, 371, 447, 539, 649, 781, 939\} kHz. The input-output signals were passed through analogue lowpass filters before the signals were sampled such that aliasing was avoided (1 MHz cutoff frequency). The measurement lasted for $T=5 \mathrm{~s}$, giving $P=5$ impedance cycles. In all, the number of samples acquired were $N=T f_{\mathrm{s}}=25 \mathrm{Ms}$ per channel (50 Ms in total).

For each measured impedance cycle, we applied the identification scheme based on the indirect local polynomial method together with the short time Fourier transform (STFT) technique described in (Sanchez et al. 2013b). The width of the STFT segment window was fixed to $60 \mathrm{~ms}$ (the integer number of periods of the noisy input-output signals processed were 60 in each block) and the segment overlap was set to $60 \%$, giving a total of 206 impedance spectra. Then, the sample mean of the BLTI impedance over the impedance cycles was computed, giving the sample mean $\widehat{Z}_{\mathrm{BLTI}}\left(j \omega_{k}\right)$. For details regarding the calibration process, we refer to Section 3.2 in (Sanchez et al. 2013a). 

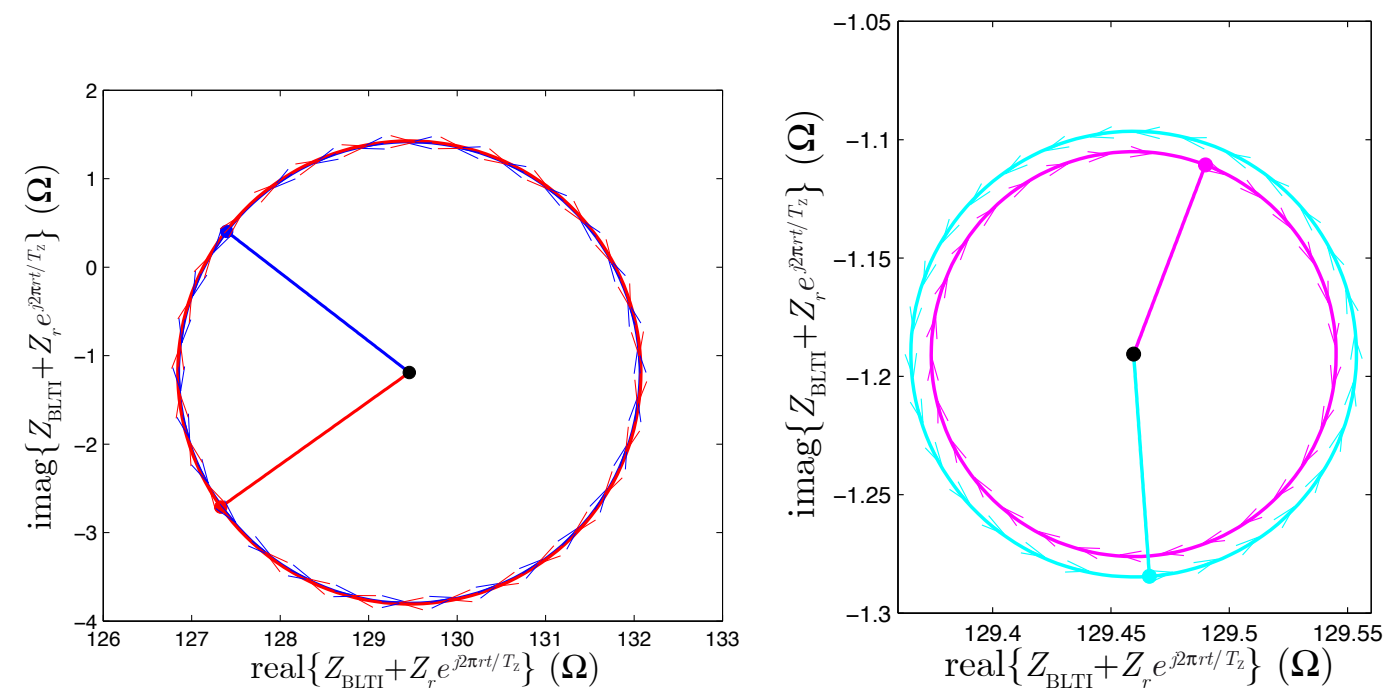

(a) $r= \pm 1, \varpi_{ \pm 1}= \pm 2 \pi \operatorname{rad~s}^{-1}$,

(b) $r= \pm 2, \varpi_{ \pm 2}= \pm 4 \pi \mathrm{rad} \mathrm{s}^{-1}$, $f_{k}=\omega_{k} / 2 \pi=1 \mathrm{kHz}$.

$f_{k}=\omega_{k} / 2 \pi=1 \mathrm{kHz}$.
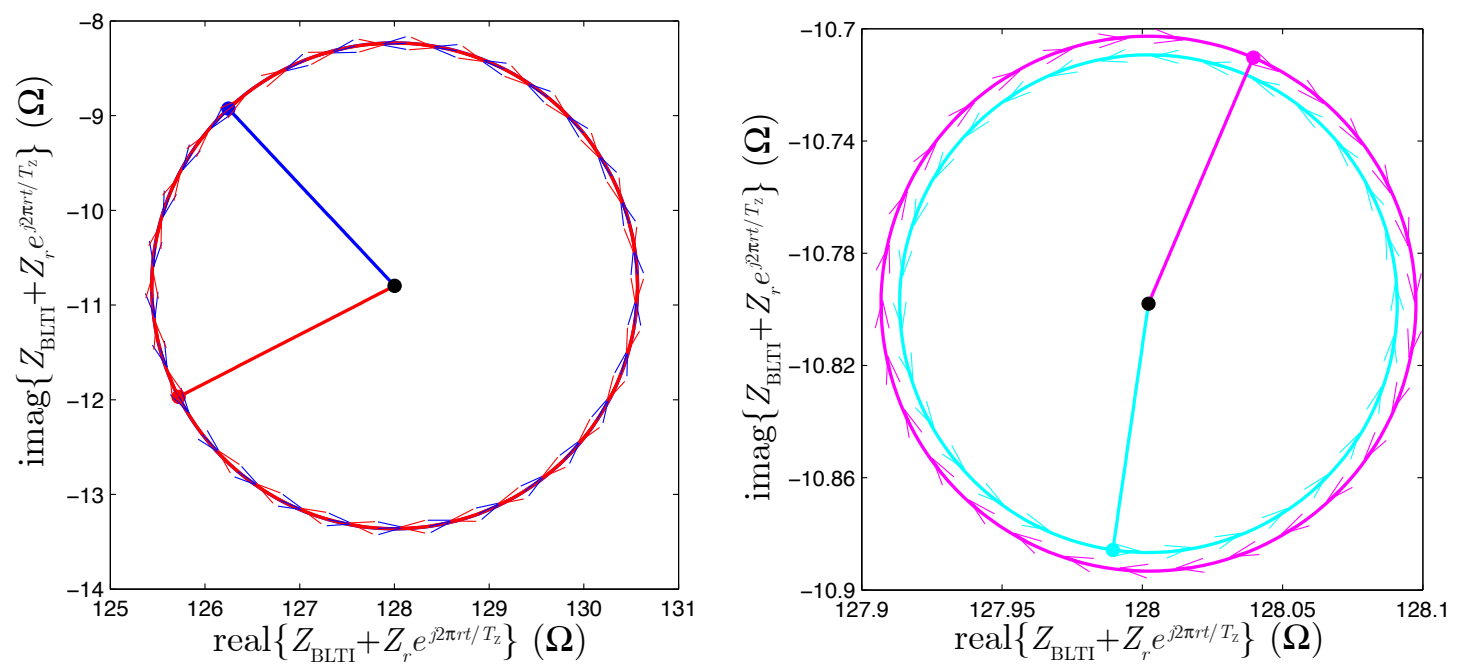

(c) $r= \pm 1, \varpi_{ \pm 1}= \pm 2 \pi \mathrm{rad} \mathrm{s}^{-1}$, $f_{k}=\omega_{k} / 2 \pi=11 \mathrm{kHz}$.

(d) $r= \pm 2, \varpi_{ \pm 2}= \pm 4 \pi \mathrm{rad} \mathrm{s}^{-1}$, $f_{k}=\omega_{k} / 2 \pi=11 \mathrm{kHz}$.

Figure 7. Circular impedance analysis of the measured periodically time-varying (PTV) impedance $Z_{\mathrm{PTV}}\left(j \omega_{k}, t\right)$ in figure 6 , with $T_{\mathrm{Z}}=1 \mathrm{~s}$ being the imposed periodicity of the impedance cycle. The black dots are the sample mean (averaged over $P=5$ impedance periods) best linear time-invariant (BLTI) impedance $\widehat{Z}_{\mathrm{BLTI}}\left(j \omega_{k}\right)$. The radii of the circles are the magnitude of the $r$ th-order harmonic impedance $\left|Z_{r}\left(j \omega_{k}\right)\right|$, $r \in \mathbb{Z}^{ \pm}$. The colored dots represent the initial phase $\Phi_{r}\left(\omega_{k}, t\right)$ at $t=0 \mathrm{~s}$. The colored arrowheads indicate the direction of rotation. The reader is referred to supplementary figure 1 to observe the time evolution of the rotating phasors. Colors: $Z_{1} e^{j \frac{2 \pi}{T} t}$ in blue, $Z_{-1} e^{-j \frac{2 \pi}{T_{\mathrm{Z}}} t}$ in red, $Z_{2} e^{j \frac{4 \pi}{T_{\mathrm{Z}}} t}$ in cyan and $Z_{-2} e^{-j \frac{4 \pi}{T_{\mathrm{Z}}} t}$ in magenta. 


\section{Results}

\subsection{2-D impedance phasor representation}

Figure 7 depicts the circular motion analysis of the PTV $R C$-circuit in figure 6 . The sample mean BLTI impedance $\widehat{Z}_{\text {BLTI }}\left(j \omega_{k}\right)$ (black dots) is the center of the circle with radius the magnitude of the $r$ th-order harmonic impedance $\left|Z_{r}\left(j \omega_{k}\right)\right|$. The initial phase (colored dots) is $\Phi_{r}\left(\omega_{k}, t\right)$ at $t=0 \mathrm{~s}$. The angular velocities for the first and second order impedance phasors are respectively $\varpi_{ \pm 1}= \pm 2 \pi \mathrm{rad} \mathrm{s}^{-1}$ (figure $7(\mathrm{a})-7(\mathrm{c})$ ) and $\varpi_{ \pm 2}= \pm 4 \pi \mathrm{rad} \mathrm{s}^{-1}$ (figure $7(\mathrm{~b})-7(\mathrm{~d})$ ). Finally, the direction of rotation is indicated with the colored arrowheads on top of the circles.

If the scale of both the real and the imaginary axis is not the same, the circle described by the impedance phasors will look like an ellipse. Note that the vertical axis, representing the reactive part of $Z_{\mathrm{BLTI}}(j \omega)+Z_{r}(j \omega) e^{j 2 \pi \frac{r}{T_{\mathrm{Z}}} t}$, is not inverted. The reason why we did not invert the $y$-axis was to keep the rotation convention as described in section 2. Otherwise, the direction of rotation would had been the opposite, i.e. counterclockwise for $r \in \mathbb{Z}^{-}$and clockwise for $r \in \mathbb{Z}^{+}$.

The strong resemblance between the positive and negative impedance phasors in figure $7(\mathrm{a})-7(\mathrm{c})$ is caused by the sinusoidal temporal variation imposed on the $R C$-circuit in figure 6 . When $Z_{\mathrm{PTV}}\left(j \omega_{k}, t\right)$ follows more or less a slow sinusoidal temporal variation, the temporal content of the low order-harmonics is distributed almost equally over the positive and negative harmonics; whereas the differences become more noticeable at high-order harmonics in figure 7(b)-7(d).

Due to the physical limitations of the circuit (shown in figure 6), the time dependence in $Z_{\mathrm{PTV}}(j \omega, t)$ does not follow a perfect sine evolution. Still, comparing the radii $\left|Z_{ \pm 1}\left(j \omega_{k}\right)\right|(\sim 2.5 \Omega)$ and $\left|Z_{ \pm 2}\left(j \omega_{k}\right)\right|(\sim 0.1 \Omega)$ in figure 7 reveals that, at these two particular frequencies of $1 \mathrm{kHz}$ and $11 \mathrm{kHz}$, the first order harmonics mostly contribute to the temporal evolution of $Z_{\mathrm{PTV}}\left(j \omega_{k}, t\right)$. In general, the purer the sinusoidal temporal variation of $Z_{\mathrm{PTV}}\left(j \omega_{k}, t\right)$ is, the lower the radii of the circumferences of the higher order harmonics (for $|r| \geq 2$ ) will be. The reader is referred to supplementary figure 1 to observe the time evolution of the rotating phasors $Z_{r}\left(j \omega_{k}\right) e^{j 2 \pi \frac{r}{T_{\mathrm{Z}}} t}$ with $r \in\{ \pm 1, \pm 2\}$. In general, the radius and initial phase of the harmonic impedance phasors will depend on the measured frequencies, as shown by the $3-\mathrm{D}$ representation in the next section.

\subsection{3-D impedance phasor representation}

Figure 8 shows the harmonic impedance phasors in figure 7 as a function of the measured frequencies (without the BLTI impedance contribution). The result is a surface of revolving phasors in the $x z$-plane. The angular velocities and the convention of rotation are the same as in figure 7 . The green line connects the initial phases $\Phi_{r}\left(\omega_{k}, t\right)$ of the harmonic impedance phasors at $t=0 \mathrm{~s}$. When the magnitude of the first order harmonic $\left|Z_{ \pm 1}\left(j \omega_{k}\right)\right|$ monotonically decreases with frequency, the surface impedance resembles a 


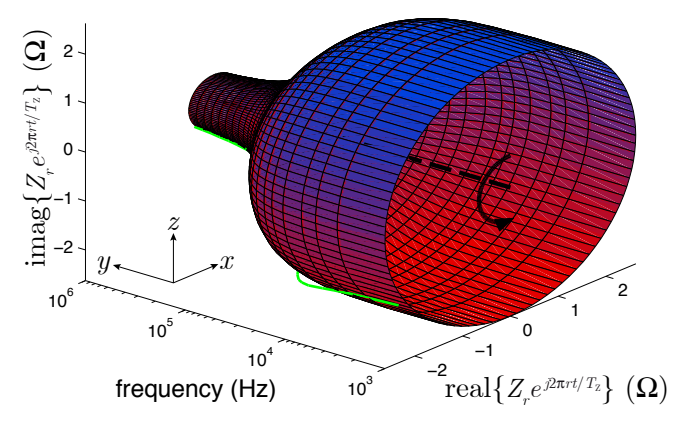

(a) $r=1, \varpi_{1}=2 \pi \operatorname{rad~s}^{-1}$.

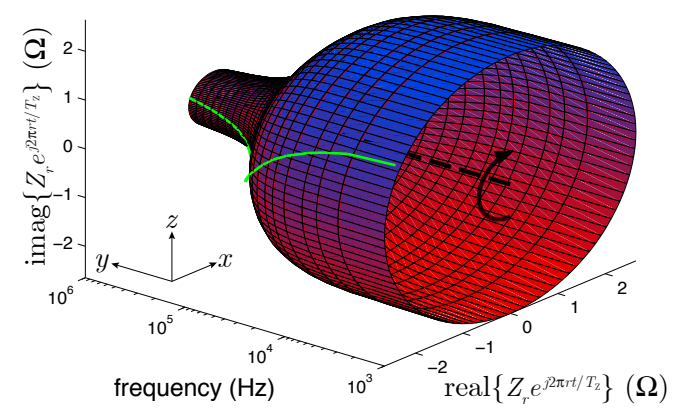

(c) $r=-1, \varpi_{-1}=-2 \pi \operatorname{rad~s}^{-1}$.

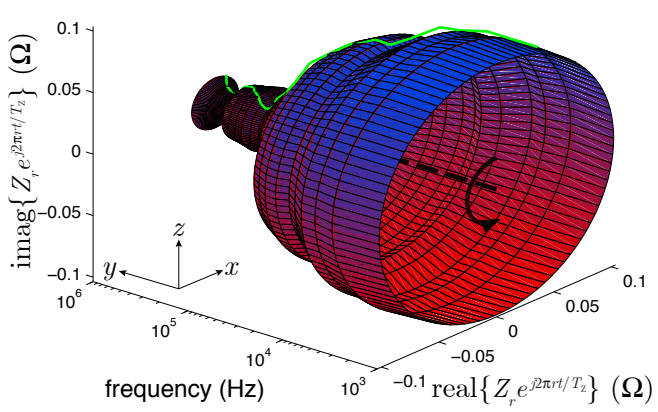

(b) $r=2, \varpi_{2}=4 \pi \operatorname{rad~s}^{-1}$.

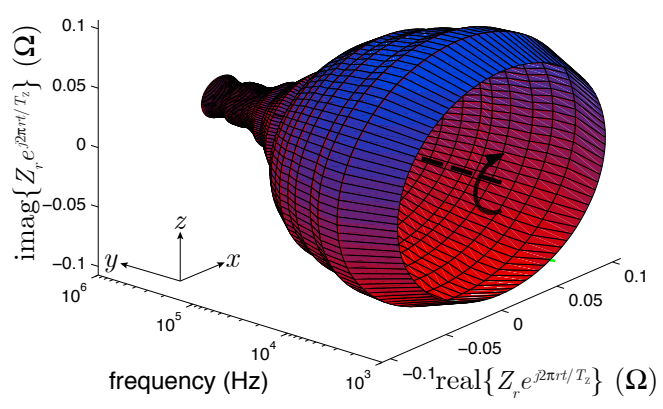

(d) $r=-2, \varpi_{-2}=-4 \pi \operatorname{rad~s}^{-1}$.

Figure 8. Three-dimensional (3-D) representation of the harmonic impedance phasors in figure 7. The result is a horn-shaped surface. The auxiliary axes of rotation are represented in each subfigure by a dashed black line (along the $y$-axis). The direction of rotation in the $x z$-plane is counterclockwise for $r \in \mathbb{Z}^{+}$and clockwise for $r \in \mathbb{Z}^{-}$. The green line connects the initial phases $\Phi_{r}\left(\omega_{k}, t\right)$ at $t=0 \mathrm{~s}$ and is represented with an offset above the surface for clarity. The reader is referred to supplementary figures 2 to 5 for an interactive 3 -D version.

horn-shaped surface, as shown in figure $8(\mathrm{a})-8(\mathrm{c})$. To grasp the details of the surface impedance in the 3-D coordinate system, we refer to supplementary figures 2 to 5 for an interactive version as well as further details.

\subsection{3-D PTV impedance representation in the Frenet-Serret frame}

We represent in figure 9 the PTV impedance in the Frenet-Serret local frame (see section 3). It can be seen that the sample mean BLTI impedance $\widehat{Z}_{\mathrm{BLTI}}\left(j \omega_{k}\right)$ (thick black line) represents an expected arc in the $y z$-plane. It is worth mentioning that, when using the Frenet-Serret frame, the units of the global $x$-axis becomes meaningless. Indeed, the $y z$-plane contains the BLTI arc (see (5)) and, moreover, only the projections on the tangent, normal, and binormal unit vectors in (6) have physical units. For clarity we left the numerical values on the $x$-axis, found through (9), to have an approximate 


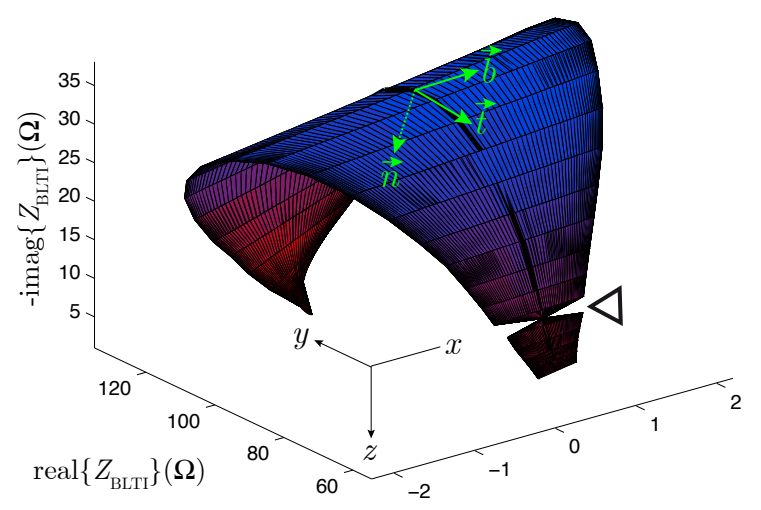

Figure 9. Three-dimensional (3-D) impedance representation of the periodically timevarying $(\mathrm{PTV})$ impedance $Z_{\mathrm{PTV}}\left(j \omega_{k}, t\right)$ in figure 6 using the Frenet-Serret local frame. The plot is originated from the vector sum of the surface impedance plots in figure $8(\mathrm{a})$ - 8 (d) torsioned by the best linear time-invariant (BLTI) impedance arc $\widehat{Z}_{\mathrm{BLTI}}\left(j \omega_{k}\right)$ (black line) (yz-plane). The flatness of the surface plot is due to the imposed sine evolution in figure 6 . The unit vectors $\vec{t}, \vec{n}, \vec{b}$ (in green) represent the tangent, normal, and binormal vectors of the Frenet-Serret frame at one particular point on the BLTI arc (black line). Although the $x$-axis does not have useful units, it is shown for convenience to indicate the strength of the temporal variation (sine evolution). The reader is referred to the supplementary figure 6 for an interactive 3-D version.

idea of the strength of the temporal variation. If, however, a scalar quantity for the degree of time-variations is desired, it can be calculated from the harmonic impedance using equation 7 in (Sanchez et al. 2015).

The flat surface behavior in figure 9 follows from the phasor plots shown in figure 7 . Since the length of the rotating phasors are similar in amplitude and the instantaneous phases are different, the geometric sum of all the rotating phasors results in a periodic straight line in the complex plane (see supplementary figure $1 \mathrm{e}, \mathrm{f}$ ). Another observation from figure 9 is the disruption of the impedance surface at high frequencies (indicated by the white arrowhead). The origin of this behavior is due to a change in sign of the normal unit vector $\vec{n}$. A subtle alteration in the curvature of $\widehat{Z}_{\text {BLTI }}\left(j \omega_{k}\right)$ happened between the measured frequencies $371 \mathrm{kHz}$ and $447 \mathrm{kHz}$. Possible error sources that can create this disruption might be a measurement error or the calibration process.

\subsection{3-D Nyquist representation}

As already mentioned in section 4, an alternative to the 3-D Frenet-Serret approach in section 6.3 is to use a 3-D Nyquist plot that takes into account the time dependency of the PTV impedance. Figure 10 shows this 3-D Nyquist representation of the PTV impedance in figure 9. One can clearly observe the imposed sinusoidal variation of the impedance from figure 6 . However, the price one pays using this representation is, in general, an increase in difficulty to immediately observe the time-invariant (mean) 


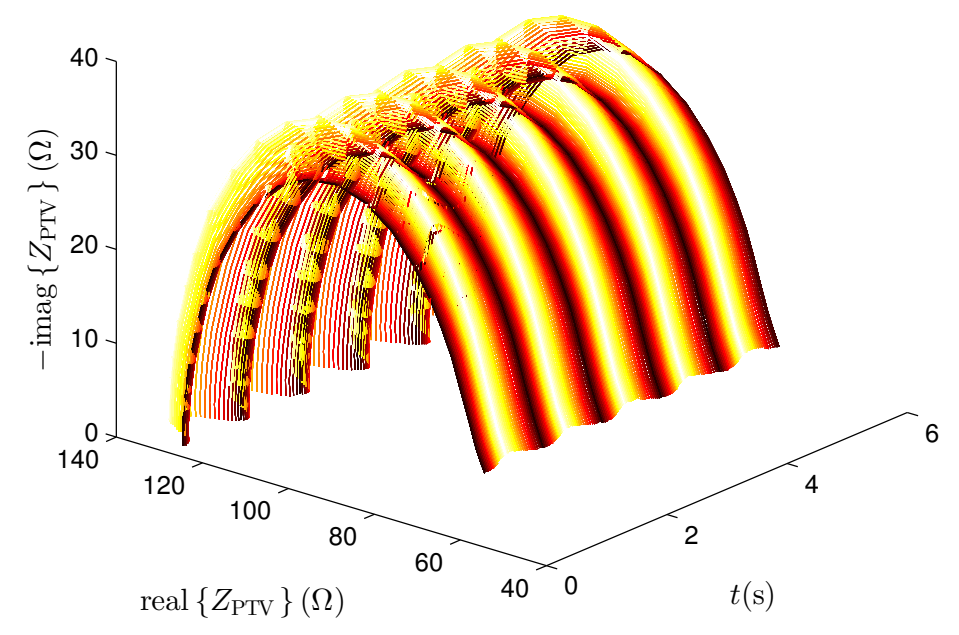

Figure 10. Three-dimensional Nyquist representation of the linear periodically timevarying (PTV) impedance $Z_{\mathrm{PTV}}(j \omega, t)$ in figure 9 (5 s, 206 impedance). The reader is referred to the supplementary figure 7 for an interactive 3 -D version.

behavior of the impedance.

\section{Discussion}

\subsection{Vector impedance analysis and 3-D Frenet-Serret approach}

From a standpoint of physical significance, both the 2-D vector impedance analysis (VIA) technique and the 3-D Frenet-Serret approach represent the same impedance. However, using the VIA approach, the time-periodic part of the impedance, $Z_{\mathrm{TP}}(j \omega, t)$ in (1), can only be represented separately from the best linear time-invariant impedance $Z_{\mathrm{BLTI}}(j \omega)$. In the VIA approach, the curvature of the BLTI arc is not taken into account. With the Frenet-Serret method, one can detect changes in the curvature of the BLTI impedance using (6)-(7). The novel representation with the Frenet-Serret $\{\vec{t}, \vec{n}, \vec{b}\}$ frame, allows us to link, at the cost of adding a new dimension, $Z_{\mathrm{BLTI}}(j \omega)$ with $Z_{\mathrm{TP}}(j \omega, t)$. 


\subsection{3-D Nyquist and Frenet-Serret representations}

The 3-D Frenet-Serret visualization method (section 3) is complementary to the wellknown 3-D Nyquist representation of $Z_{\mathrm{PTV}}(j \omega, t)$. Basically, they represent the same impedance but they both do it in a different way. The 3-D Nyquist representation is formed by the instantaneous impedance values of $Z_{\mathrm{PTV}}(j \omega, t)$ at different instants of time, while in the 3-D Frenet-Serret approach the time-periodic part is shaped by the curvature of the BLTI impedance arc.

If, for instance, one is interested in analyzing the mean (time-invariant) dynamics of $Z_{\mathrm{PTV}}(j \omega, t)$, it can be quite burdensome using the 3-D Nyquist representation. However, with the new 3-D Frenet-Serret approach, it can be read straightforwardly from the curvature of the plot. This is found to be very important because it allows us to analyze the mean dynamic behavior of $Z_{\mathrm{PTV}}(j \omega, t)$ using time-invariant equivalent electrical circuits and models without penalizing the representation of the temporal evolution.

On the other hand, it is easier to see the time evolution of $Z_{\mathrm{PTV}}(j \omega, t)$ using the 3-D Nyquist visualization than by looking at the 3-D Frenet-Serret plot where the time axis is lost. From a practical point of view, the 3-D Nyquist plot it is easier to represent and is valid for any kind of temporal impedance evolution. Whereas the 3-D Frenet-Serret method presented here is applied to time-varying impedances with a dominant periodic character such as breathing and cardiac activities.

Finally, the 3-D Frenet-Serret plot is based on a parametric-in-time impedance measurement (all the frequencies are measured at the same time instant continuously over time) (Sanchez et al. 2013a). Therefore, it cannot be used in stepped-sine IS experiments where each frequency is measured at a different time instant, that not being a limitation with the 3-D Nyquist plot.

\section{Conclusions}

In this paper, we have shown that, when a time-variant impedance has a dominant timeperiodic dependence, the temporal evolution can be analyzed using the circular motion analysis technique. Once the frequency dependence is included, the revolution of the impedance phasors forms a surface in a three-dimensional (3-D) space. This idea gave rise to a new $3-\mathrm{D}$ visualization tool based on the Frenet-Serret local frame. The method takes properly into account the curvature of the mean behavior of the periodically varying impedance. This $3-\mathrm{D}$ Frenet-Serret approach is then compared with the 3-D extension of the classical 2-D Nyquist representation, which is used frequently in the literature to interpret linear time-invariant impedance. The novel visualization tool might help to disentangle multiple simultaneous physiological processes with temporal varying dynamics. 
Circular motion analysis of time-varying bioimpedance

\section{Author contributions}

B.S and E.L were the main authors of the manuscript. Conceived and designed the experiments: B.S E.L. Performed the experiments: B.S. Analyzed the data: B.S E.L. Wrote the paper: B.S E.L. Revised the manuscript: R.P. Approved the manuscript: S.R R.P.

\section{Acknowledgments}

This work has been supported by the Fund for Scientific Research (FWO- Vlaanderen), by the Flemish Government (Methusalem METH1), and by the Belgian Government through the Interuniversity Attraction Poles (IAP VII, Dysco) Program.

\section{References}

Allen J \& Rabiner L 1977 Proc. IEEE 65(11), 1558-1564.

Barsoukov E \& Macdonald J R 2005 Impedance Spectroscopy: Theory, Experiment, and Applications 2nd edn Wiley-Interscience.

Bernstein D P \& Lemmens H J M 2005 Medical \& Biological Engineering \& Computing 43(4), 443-450.

Eisenberg R S 1980 in 'Handbook of Physiology, Section 10: Skeletal Muscle' John Wiley \& Sons, Inc. pp. 301-323.

Frenet F 1852 Journal de Mathématiques Pures et Appliquées 17, 437-447.

Grimnes S \& Martinsen O G 2014 Bioimpedance and Bioelectricity Basics 3rd edn Academic Press.

Kubicek W G, Karnegis J N, Patterson R P, Witsoe D A \& Mattson R H 1966 Aerospace medicine 37(12), 1208-12.

Louarroudi E, Lataire J, Pintelon R, Janssens P \& Swevers J 2013 Mechanical Systems and Signal Processing pp. 1-25.

Louarroudi E, Pintelon R \& Lataire J 2012 IEEE Transactions on Instrumentation and Measurement 61(5), 1384-1394.

Louarroudi E, Pintelon R \& Lataire J 2014 Mechanical Systems and Signal Processing 48(1-2), 274-299.

Niedzwiecki M \& Kaczmarek P 2005 IEEE Trans. Signal Process. 53(12), 4588-4598.

Pintelon R, Louarroudi E \& Lataire J 2015 IEEE Trans. Instrum. Meas. pp. 1-1.

Pintelon R \& Schoukens J 2012 System Identification: A Frequency Domain Approach 2nd edn IEEE Press.

Pop V, Bergveld H J, Notten P H L \& Regtien P P L 2005 Meas. Sci. Technol. 16(12), R93-R110.

Sams A \& Marmarelis V 1988 Automatica 24(4), 563-567.

Sanchez B, Louarroudi E, Bragos R \& Pintelon R 2013b Physiological measurement 34(10), 1217-1238.

Sanchez B, Louarroudi E, Jorge E, Cinca J, Bragos R \& Pintelon R 2013a Physiological measurement 34(3), 339-57.

Sanchez B, Louarroudi E \& Pintelon R 2015 Physiological Measurement 36(3), 595-620.

Sanchez B, Vandersteen G, Bragos R \& Schoukens J 2012 Measurement Science and Technology 23(10), 105501.

Searle A \& Kirkup L 1999 Physiological Measurement 20(1), 103-114.

Serret J A 1851 Journal de Mathématiques Pures et Appliquées 16, 193--207.

Sherwood A, Allen M T, Fahrenberg J, Kelsey R M, Lovallo W R \& van Doornen L J 1990 Psychophysiology 27(1), 1-23.

Tóth R, Laurain V, Gilson M \& Garnier H 2012 Automatica 48(9), 2314-2320.

Van Ingelgem Y, Tourwé E, Vereecken J \& Hubin A 2008 Electrochim. Acta 53(25), 7523-7530.

Zadeh L A 1950 a Proceedings of the IRE 38(3), 291-299. 
Zadeh L A 1950 b Journal of Applied Physics 21(7), 642. 